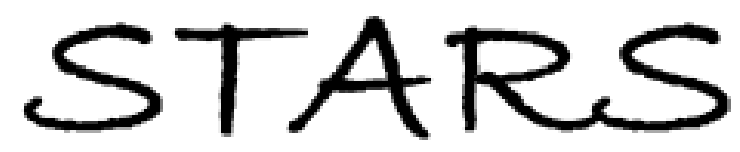

University of Central Florida

STARS

$1-1-2013$

\title{
Coherent coupling between exciton resonances governed by the disorder potential
}

Yuri D. Glinka

Zheng Sun

Mikhail Erementchouk

University of Central Florida

Michael N. Leuenberger

University of Central Florida

Alan D. Bristow

See next page for additional authors

Find similar works at: https://stars.library.ucf.edu/facultybib2010

University of Central Florida Libraries http://library.ucf.edu

This Article is brought to you for free and open access by the Faculty Bibliography at STARS. It has been accepted for inclusion in Faculty Bibliography 2010 s by an authorized administrator of STARS. For more information, please contactSTARS@ucf.edu.

\section{Recommended Citation}

Glinka, Yuri D.; Sun, Zheng; Erementchouk, Mikhail; Leuenberger, Michael N.; Bristow, Alan D.; Cundiff, Steven T.; Bracker, Allan S.; and Li, Xiaoqin, "Coherent coupling between exciton resonances governed by the disorder potential" (2013). Faculty Bibliography 2010s. 4034.

https://stars.library.ucf.edu/facultybib2010/4034

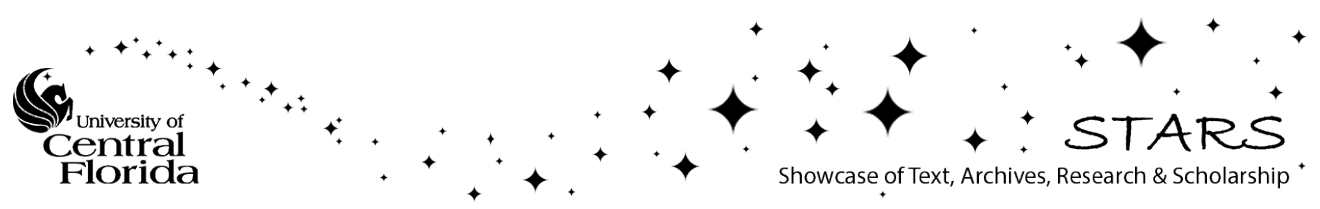




\section{Authors}

Yuri D. Glinka, Zheng Sun, Mikhail Erementchouk, Michael N. Leuenberger, Alan D. Bristow, Steven T. Cundiff, Allan S. Bracker, and Xiaoqin Li 


\title{
Coherent coupling between exciton resonances governed by the disorder potential
}

\author{
Yuri D. Glinka, ${ }^{1,2}$ Zheng Sun, ${ }^{1}$ Mikhail Erementchouk, ${ }^{3}$ Michael N. Leuenberger, ${ }^{3}$ Alan D. Bristow, ${ }^{4,5}$ Steven T. Cundiff, ${ }^{4}$ \\ Allan S. Bracker, ${ }^{6}$ and Xiaoqin $\mathrm{Li}^{1, *}$ \\ ${ }^{1}$ Department of Physics, University of Texas, Austin, Texas 78712, USA \\ ${ }^{2}$ Institute of Physics, National Academy of Sciences of Ukraine, Kiev, 03028, Ukraine \\ ${ }^{3}$ NanoScience Technology Center and Department of Physics, University of Central Florida, Orlando, Florida 32826, USA \\ ${ }^{4}$ JILA, University of Colorado and National Institute of Standard and Technology, Boulder, Colorado 80309-0440, USA \\ ${ }^{5}$ Department of Physics and Astronomy, West Virginia University, Morgantown, West Virginia 26506-6315, USA \\ ${ }^{6}$ Naval Research Lab, Washington, DC 20375, USA
}

(Received 3 October 2012; revised manuscript received 22 July 2013; published 27 August 2013)

\begin{abstract}
Monolayer fluctuations in the thickness of a semiconductor quantum well $(\mathrm{QW})$ lead to the formation of spectrally resolved excitons located in the narrower, average, and thicker regions of the QW. Whether or not these excitons are coherently coupled via Coulomb interaction is a long-standing debate. We demonstrate that different types of disorder potential govern coherent coupling among excitons, and the coupling strength can be quantitatively measured using optical two-dimensional Fourier transform spectroscopy. Strong coherent coupling occurs between certain types of excitons but is missing between other types of excitons because the distinctive nature of excitons results in different spatial overlap. Our finding may be applicable to other disordered systems, such as photosynthesis and conjugated polymers, where exciton coupling plays a critical role in determining charge and energy transfer.
\end{abstract}

DOI: 10.1103/PhysRevB.88.075316

PACS number(s): 78.67.De, 42.50.Hz, 81.07.Ta

\section{INTRODUCTION}

Disorder at surfaces and interfaces plays an increasingly important role in ever-shrinking electronic devices. Even in nanostructures of the highest quality, monolayer fluctuations are inevitable. Importantly, monolayer fluctuations at interfaces of quantum wells (QWs) change the QW thickness, leading to wider or narrower regions referred to as widerwidth (WW) disorder and narrower-width (NW) disorder, respectively. Under optical excitation, bound electron-hole pairs (excitons) that form in such QWs are governed by the disorder potential and will often exhibit several spectrally distinct resonances, ${ }^{1-4}$ instead of a single inhomogeneously broadened resonance. Whether or not these different types of excitons are coherently coupled via Coulomb interactions is an outstanding and much debated question. ${ }^{5-9}$ Previous investigations have yielded conflicting results partially due to the limited spectral and temporal information accessible using traditional spectroscopic methods and partially due to insufficient control of the disorder at the QW interfaces.

Understanding coherent interaction among multiple electronic states is a prerequisite to controlling material properties at the level of electrons and is a challenge that is ubiquitous in material science. Specifically, the presence or absence of coherent coupling among spectrally resolved excitons significantly influence energy transfer, ${ }^{10}$ photon emission statistics, ${ }^{11}$ and even quantum logic operations ${ }^{12}$ in semiconductor heterostructures such as QWs, quantum wires, and quantum dots. This problem is also relevant for a broader range of materials, including natural/artificial photosynthetic systems ${ }^{13-15}$ and conjugated polymers. For example, exciton dissociation in conjugated polymers occurs in a two-step process: the formation of a charge-transfer state at an energy level above the initial exciton followed by a complete dissociation process. ${ }^{16,17}$ The formation of the charge-transfer state is dictated by the interplay of the disorder and the
Coulomb interaction, which is the essence to the problem of interest here.

In this paper, we investigate coherent coupling between different types of excitons in a single, narrow GaAs/AlGaAs QW using the powerful technique of optical two-dimensional (2D) Fourier transform spectroscopy (2DFTS). ${ }^{18-23}$ We first identify three types of excitons confined in different regions of the QW. We refer to the excitons confined in the wider, average-thickness, and narrower regions of the $\mathrm{QW}$ as type $A$, $B$, and $C$ excitons, respectively, as illustrated in Fig. 1(a). Using 2DFTS, we are able to separate complex quantummechanical pathways in the coherent nonlinear response and unambiguously identify strong coherent coupling between type $B$ and $C$ excitons. In contrast, such coupling is missing between type $A$ and $B$ excitons. This difference in exciton coupling originates from the nature of exciton resonances governed by the disorder potential: type $A$ excitons are bound states, mainly localized within the WW disorder potential; type $B$ are delocalized in the average thickness regions; and type $C$ excitons are scattering resonances associated with NW defects. Different spatial overlap between different types of excitons is responsible for the absence or presence of coupling in the 2D spectra. This conclusion is supported by calculations based on a single-defect model.

\section{EXPERIMENTS}

2DFTS is a heterodyne-detected four-wave mixing (FWM) technique, which monitors and correlates nonlinear polarization phase evolution during two independent periods, $\tau$ and $t$, separated by a waiting period $T$, as illustrated in Fig. 1(b). A $2 \mathrm{D}$ spectrum as a function of the absorption frequency $\omega_{\tau}$ and emission frequency $\omega_{t}$ is then obtained by Fourier transforming the FWM signal with respect to time variables $\tau$ and $t$. A peak in a $2 \mathrm{D}$ spectrum indicates that an oscillation at absorption frequency $\omega_{\tau}$ during the first time period gives rise 


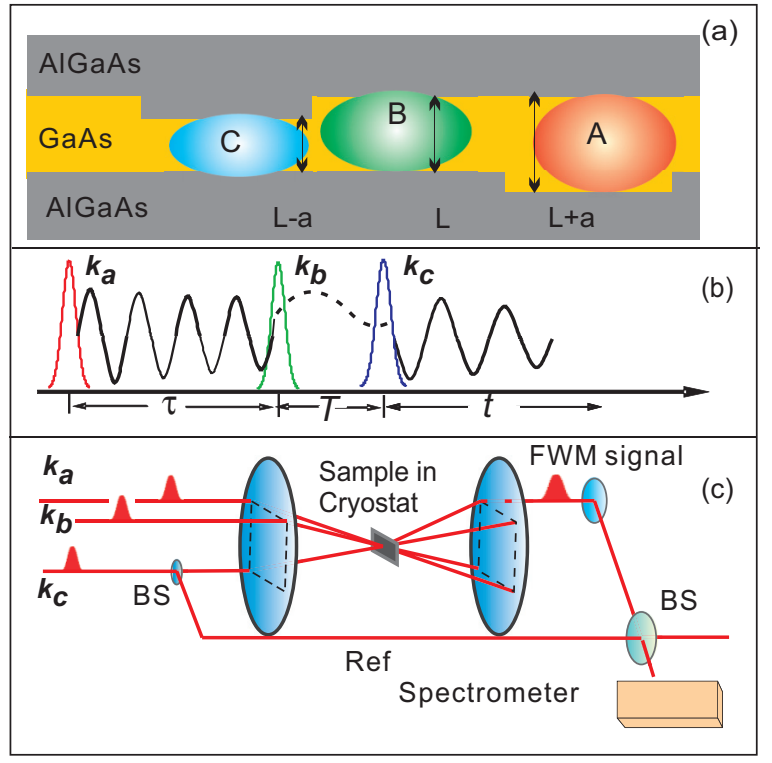

FIG. 1. (Color online) (a) Illustration of different types of excitons in a disordered QW. (b) Pulse sequence and (c) experimental setup in 2DFTS.

to an oscillation at emission frequency $\omega_{t}$ during the third period. Coupling between resonances can be identified by the presence of cross peaks in 2D spectra, for which $\omega_{\tau} \neq$ $\omega_{t}$. 2DFTS is particularly suitable for quantifying coupling among multiple electronic transitions, as quantum-mechanical pathways associated with coupling are isolated in the spectra.

The experimental setup is described in detail elsewhere. ${ }^{24}$ Briefly, three phase-stabilized, collinearly polarized excitation pulses with wave vectors $\boldsymbol{k}_{a}, \boldsymbol{k}_{b}$, and $\boldsymbol{k}_{c}$ are arranged in box geometry, generating a complex FWM signal in the phase-matched direction: $\boldsymbol{k}_{s}=-\boldsymbol{k}_{a}+\boldsymbol{k}_{b}+\boldsymbol{k}_{c}$, as illustrated in Fig. 1(c). The excitation pulse sequence chosen is the rephasing time ordering, where the conjugate pulse $\left(\boldsymbol{k}_{a}\right)$ arrives first, and $\boldsymbol{k}_{c}$ arrives last. The evolution time $(\tau)$ between $\boldsymbol{k}_{a}$ and $\boldsymbol{k}_{b}$ pulses, the waiting time $(T)$ between pulses $\boldsymbol{k}_{b}$ and $\boldsymbol{k}_{c}$, and the emission time $(t)$ after the arrival of pulse $\boldsymbol{k}_{c}$ govern the complex signal, $S(\tau, T, t)$. We resolve the phase information of the signal field, which is made possible by heterodyne detection with a phase-stabilized reference beam and stepping the excitation pulse delays with interferometric precision. The emission frequency $\left(\omega_{t}\right)$ is determined by sending the signal through a spectrometer, and the absorption frequency $\left(\omega_{\tau}\right)$ is retrieved by a numerical Fourier transform of the $S\left(\tau, T, \omega_{t}\right)$ data with respect to $\tau$.

We studied a series of $\mathrm{GaAs} / \mathrm{Al}_{0.3} \mathrm{Ga}_{0.7} \mathrm{As}$ single QW's with four different thickness grown by molecular beam epitaxy on a GaAs(100) substrate. The nominal thicknesses are $4.2,6.2,8.4$, and $14.0 \mathrm{~nm}$, respectively. Two-minute growth interruption at the interfaces results in monolayerwidth fluctuations with relatively large lateral dimensions of the order of tens of nanometers. The sample was held at $4.2 \mathrm{~K}$ in optical experiments. The peak excitation power density from 7 to $11.5 \mathrm{MW} / \mathrm{cm}^{2}$ was used, corresponding to an estimated sheet exciton density $\sim 0.5 \times 10^{10} \mathrm{~cm}^{-2}$. At this high excitation power, one expects excitation-induced

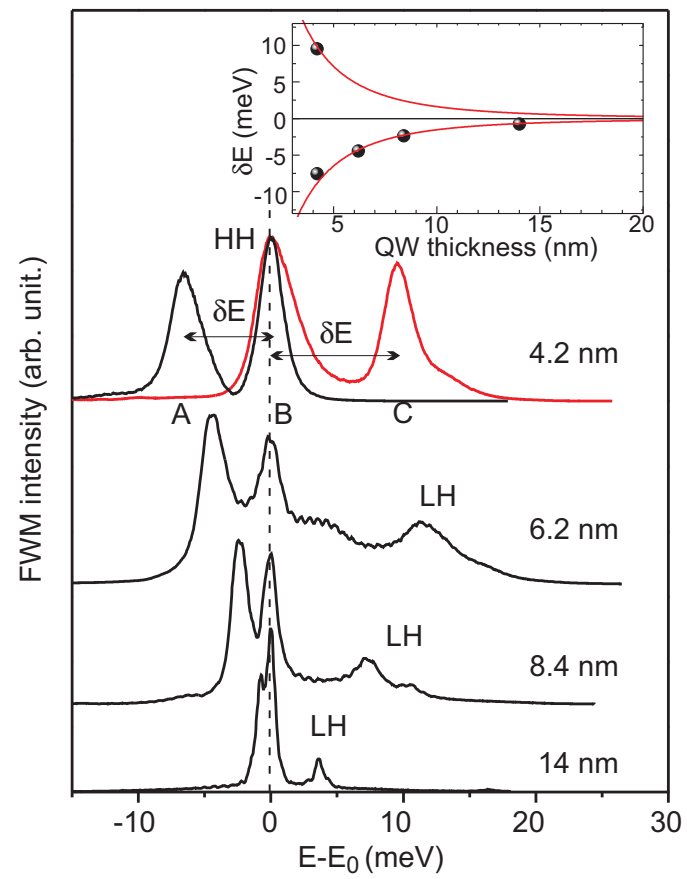

FIG. 2. (Color online) FWM spectra plotted with relative photon energy $\left(E-E_{0}\right)$, where $E_{0}$ specifies the position of $\mathrm{HH}$ excitons residing in the regions of the perfect QW. Two spectra from the 4.2-nm QW are taken for different laser tuning. Inset: QW width dependence of the $\mathrm{HH}$ exciton resonance splitting due to the monolayer thickness fluctuations at the interface (dots) relative to $B$-type excitons. The red lines present the result of theoretical fits.

dephasing ${ }^{25,26}$ to contribute significantly to the linewidth of the exciton resonances.

We first identify relevant exciton resonances via FWM spectra displayed in Fig. 2. The heavy-hole (HH) and light-hole (LH) excitons are split by confinement in the growth direction in a QW. The LH excitons are clearly observed and labeled for QWs with nominal thickness of 6.2, 8.4, and $14 \mathrm{~nm}$. The LH exciton in the $4.2 \mathrm{~nm}$ QW is shifted further to the higher energy, outside the excitation bandwidth. We will focus on $\mathrm{HH}$ resonances in the current study.

Close inspection reveals that $\mathrm{HH}$ resonances are split into two or three resonances, which arise from the monolayer fluctuations of the QW thickness. This assignment can be proven by investigating the systematic change of the monolayer splitting as a function of the average $\mathrm{QW}$ width. We initially make the reasonable assumption that the lowest energy peak originates from type $A$ excitons residing in the WW disorder potential. This allows us to plot the FWM spectra as a function of the relative energy $\delta E=\left(E-E_{0}\right)$, where $E_{0}$ specifies the energy for type $B$ excitons in the perfect $\mathrm{QW}$. Negative $\delta E$ corresponds to type $A$ excitons residing in WW disorders, while positive $\delta E$ corresponds to type $C$ excitons formed near NW disorder. We then plot $\delta E$ as a function of the nominal QW thickness in the inset of Fig. 2. Within the effective mass approximation, $\delta E$ due to monolayer fluctuation of the QW width $\left(\delta L^{*}= \pm a\right)$ is

$$
\delta E=\frac{h^{2} \pi^{2}}{2 \mu_{H H}}\left(\frac{1}{\left(L^{*} \pm a\right)^{2}}-\frac{1}{L^{* 2}}\right) \approx-\frac{h^{2} \pi^{2} \delta L^{*}}{\mu_{H H} L^{* 3}},
$$


where $L^{*}=L+2 \Lambda$ accounts for an enlargement of the QW width owing to an average wave $=$ function penetration depth into the $\mathrm{Al}_{0.3} \mathrm{Ga}_{0.7} \mathrm{As}$ barrier $(\Lambda=1.5 \mathrm{~nm}) .{ }^{27}$ We first extracted the in-plane reduced exciton mass $\mu_{\mathrm{HH}}=0.055 m_{\mathrm{e}}$ from the dependence of $E_{0}$ on the QW width (not shown explicitly). We then fitted the data in Fig. 2 using Eq. (1) to obtain $a=0.25 \mathrm{~nm}$, which matches the thickness of one atomic monolayer in GaAs. This analysis as well as previous experiments supports our assignment that these resonances correspond to excitons localized in regions with monolayer thickness difference. The energy splitting between these resonances is larger than that expected from excitonic excited states ${ }^{28}$ or trions ${ }^{29}$ (see Supplemental Material in Ref. 30). We focus the rest of the paper on 2DFTS experiments performed on the 4.2-nm-thick QW, as it clearly exhibited all three types of $\mathrm{HH}$ excitons. ${ }^{31}$ The probability of forming a type $C$ exciton is reduced in wider QWs.

The laser bandwidth is $\sim 16 \mathrm{meV}$; hence, two $2 \mathrm{D}$ spectra were acquired with the laser tuned to optimally excite two of the three $\mathrm{HH}$ exciton resonances at one time. The waiting time was set to be $T=125$ fs to avoid temporal pulse overlap. Figure 3 shows the normalized 2D amplitude spectra together with the corresponding regular FWM and laser spectra. We did not observe clear evidence of coupling between exciton $A$ and $B$ in Fig. 3(b). In contrast, strong coherent coupling between exciton resonance $B$ and $C$ is identified as the cross-peak $B C$ in Fig. 3(d). One may expect another cross peak at the location indicated by $C B$. Previous work has documented that 2D spectra of semiconductor QWs display a strong asymmetry in amplitude between cross peaks, ${ }^{32}$ which explains the absence of a clear cross peak at location indicated by $C B$.

Prior experiments aimed at investigating coherent coupling among different types of excitons only permitted one to look for qualitative signatures, such as oscillatory behavior in time-resolved or spectrally resolved FWM experiments. ${ }^{5,6}$ The interpretation of these experiments is challenging, due to the limited spectral or temporal information accessible to conventional spectroscopy methods. In contrast, 2DFTS

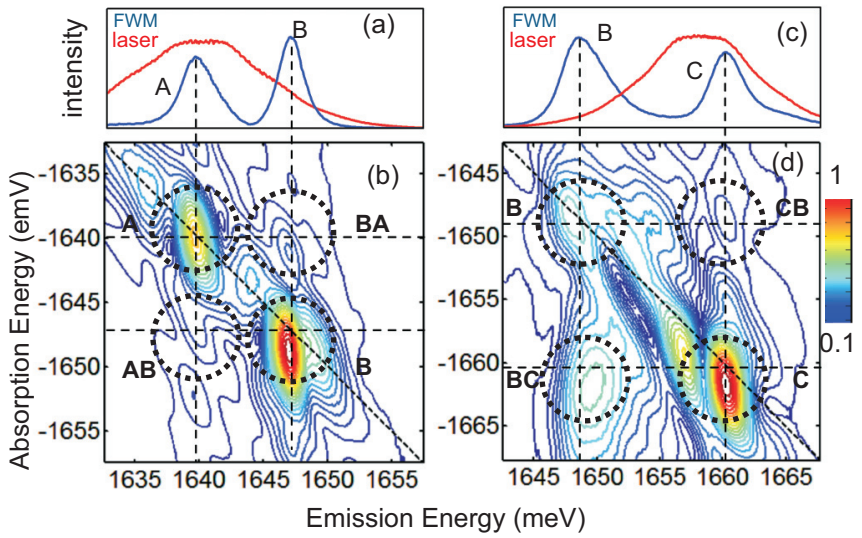

FIG. 3. (Color online) FWM and normalized 2DFTS amplitude spectra taken on the $4.2-\mathrm{nm} \mathrm{QW}$ at $T=125 \mathrm{fs}$, with colinearly polarized excitation beams (a) and (b), when exciton $A$ and $B$ resonances are excited, and (c) and (d), when exciton $B$ and $C$ resonances are excited. allows us to quantify the strength of coherent coupling between these resonances, rather than only asking whether such coupling exists. In the case of a $V$-level system, for example, a strong coherent coupling induced by a common electronic state should lead to the maximum cross-peak intensity to be the geometric average of the two diagonal peak intensities. ${ }^{33}$ In Fig. 3(d), the coupling between resonances $B$ and $C$ is quantified by the ratio between the cross-peak $B C$ and the geometric average of the diagonal peak intensities, giving a value of $\sim 0.6$ that is significantly above the noise level. In contrast, we observe no clear cross peaks in Fig. 3(b). We place an upper bound for the coupling between resonances $A$ and $B$ to be below 0.1 , limited by the noise level in the 2D spectra. The strong cross-peak intensity $B C$ at the chosen $T$ suggests that it arises from electronic coherent coupling and not population relaxation. We confirm that the lifetimes of the excitons are beyond tens of picoseconds with spectrally and temporally resolved pump-probe measurements (see Supplemental Material in Ref. 30). The slow relaxation process cannot account for the strong cross-peak $B C$ in the $2 \mathrm{D}$ spectra [Fig. 3(d)] at the short waiting time. Incoherent coupling due to population relaxation between excitons confined in different thickness regions of a $\mathrm{QW}$ has been investigated previously using 2DFTS and observed at waiting times of 20 ps or longer. ${ }^{34}$ Another recent experiment conducted on individual excitons localized in a QW presented evidence of coherent coupling in certain regions of the sample, ${ }^{9}$ possibly consistent with our experimental findings.

We briefly comment on other features of the 2D spectra, which are not essential for identifying the coupling or quantifying the coupling strength. There is a peak near the diagonal peak $C$ in Fig. 3(d), which may be due to the formation of biexcitons investigated in other recent 2DFTS experiments. ${ }^{35-37}$ There are also small satellite peaks near the diagonal line that arise from the direct scattering of excitation pulses. $^{24}$ These spurious peaks do not contaminate the measurements of the cross peaks. Thus, no error was introduced in the quantitative evaluation of the coupling strength. Finally, the line shapes of the peaks (especially the diagonal peaks) are elongated along the absorption frequency axis. We attribute the elongation to the relatively high laser power used in the experiments, and it may be related to the excitation-induced dephasing.

\section{THEORETICAL MODEL}

In order to interpret the measured 2D spectra, we perform calculations based on microscopic theory of semiconductor coherent nonlinear response in QWs with in-plane inhomogeneous disorder potentials. ${ }^{38,39}$ Coherent semiconductor excitations are described by exciton operators

$$
B_{\mu}^{+}=\sum_{\sigma_{\mu}} \int d \boldsymbol{x}_{1} d \boldsymbol{x}_{2} \phi_{\mu}\left(\boldsymbol{x}_{1}, \boldsymbol{x}_{2}\right) v_{\sigma_{\mu}}^{+}\left(\boldsymbol{x}_{2}\right) c_{s_{\mu}}^{+}\left(\boldsymbol{x}_{1}\right),
$$

where $c_{s}^{+}$and $v_{\sigma}^{+}$are the operators creating the electron with spin $s$ at point $\boldsymbol{x}_{1}$ and the hole with spin $\sigma$ at point $\boldsymbol{x}_{2}$, respectively, and we have introduced the combined notation for the spin state of the electron-hole pair $\sigma=\{s, \sigma\}$. 
The functions $\phi_{\mu}\left(\boldsymbol{x}_{1}, \boldsymbol{x}_{2}\right)$ are chosen in such a way that operators $B_{\mu}^{+}$create genuine single electron-hole pair states: $H_{S C} B_{\mu}^{+}|0\rangle=E_{\mu}|0\rangle$, where $|0\rangle$ is the semiconductor ground state (i.e., empty conductance and filled valence bands) and $H_{S C}$ is the semiconductor Hamiltonian without the external field.

The existence of hierarchy of characteristic energy scales governing the electron dynamics together with the assumption that the most important contribution results from the lowest energy states is employed by the well-known ansatz (see e.g., Refs. 1, 40)

$$
\phi_{\mu}\left(\boldsymbol{x}_{1}, \boldsymbol{x}_{2}\right)=u_{e}\left(z_{1}, \boldsymbol{\rho}_{1}\right) u_{\sigma_{\mu}}\left(z_{2}, \boldsymbol{\rho}_{2}\right) \psi_{\sigma}\left(\boldsymbol{\rho}_{1}-\boldsymbol{\rho}_{2}\right) \Phi_{\mu}(\boldsymbol{R}) .
$$

Here, the functions $u_{e, \sigma}(z, \rho)$ describe the local confinement of the electrons and holes in the growth direction, $z$, at point $\rho$ of the QW, $\psi_{\sigma}(\rho)$ is the wave function of the relative motion of $2 d$ electrons and holes, and $\Phi_{\mu}(\boldsymbol{R})$ is the wave function of the exciton center of mass. This approximation can be used for introducing the operators of the exciton center of mass $B_{\sigma}^{+}(\boldsymbol{R})$ by representing

$$
B_{\mu}^{+}=\int d \boldsymbol{R} \Phi_{\mu}(\boldsymbol{R}) B_{\sigma_{\mu}}^{+}(\boldsymbol{R}),
$$

with

$$
B_{\sigma}^{+}(\boldsymbol{R})=\int d z_{1} d z_{2} d \rho u_{e}\left(x_{1}\right) u_{\sigma}\left(x_{2}\right) \psi_{\sigma}(\rho) v_{\sigma}^{+}\left(x_{2}\right) c_{s}^{+}\left(x_{1}\right) \text {, }
$$

where $\quad \boldsymbol{x}_{1}=z_{1} \hat{z}+\boldsymbol{R}+\rho m_{e} / M_{\sigma} \quad$ and $\quad \boldsymbol{x}_{2}=z_{2} \hat{z}+\boldsymbol{R}-$ $\rho m_{\sigma} / M_{\sigma}$ with $\hat{z}$ being the unit vector along the $z$ direction, and $m_{e}, m_{\sigma}$ and $M_{\sigma}=m_{e}+m_{\sigma}$ being electron, hole, and exciton masses, respectively.

Using this representation, the equation of motion for the nonlinear polarization is found within the $\chi^{(3)}$ approximation $^{41-44}$ to be

$$
\left(i \frac{\partial}{\partial t}+i \gamma_{\sigma}-\hat{H}_{\sigma}(\boldsymbol{R})\right) P_{\sigma}^{(3)}(\boldsymbol{R}, t)=F_{\sigma}^{(3)}(\boldsymbol{R}, t) .
$$

In order to reproduce the qualitative features of $2 \mathrm{D}$ spectra, we can neglect the effect of Pauli blocking and invoke the short-memory approximation ${ }^{38,45}$ to account for the Coulomb correlation. Under these approximations, the driving term is

$$
\begin{aligned}
F_{\sigma}^{(3)}(\boldsymbol{R}, t)= & -\frac{1}{2} \sum_{\sigma_{1}, \boldsymbol{\sigma}_{2}, \sigma_{3}} \int d \boldsymbol{R}_{1,2,3} \beta_{\sigma, \sigma_{1}}^{\sigma_{2}, \sigma_{3}}\left(\boldsymbol{R}, \boldsymbol{R}_{1}, \boldsymbol{R}_{2}, \boldsymbol{R}_{3}\right) \\
& \times p_{\sigma_{1}}^{*}\left(\boldsymbol{R}_{1}, t\right) p_{\sigma_{2}}\left(\boldsymbol{R}_{2}, t\right) p_{\sigma_{3}}\left(\boldsymbol{R}_{3}, t\right),
\end{aligned}
$$

where

$$
\begin{aligned}
\beta_{\sigma, \sigma_{1}}^{\sigma_{2}, \sigma_{3}}\left(\boldsymbol{R}, \boldsymbol{R}_{1}, \boldsymbol{R}_{2}, \boldsymbol{R}_{3}\right) \\
=\left\langle D_{\boldsymbol{\sigma}, \sigma_{1}}\left(\boldsymbol{R}, \boldsymbol{R}_{1}\right) B_{\sigma_{2}}^{+}\left(\boldsymbol{R}_{2}\right) B_{\sigma_{3}}^{+}\left(\boldsymbol{R}_{3}\right)\right\rangle \\
\quad+\frac{i}{\gamma_{\sigma_{2}}+\gamma_{\sigma_{3}}}\left\langle D_{\sigma, \sigma_{1}}\left(\boldsymbol{R}, \boldsymbol{R}_{1}\right) D_{\sigma_{2}, \sigma_{3}}^{+}\left(\boldsymbol{R}_{2}, \boldsymbol{R}_{3}\right)\right\rangle,
\end{aligned}
$$

where $D_{\sigma, \sigma_{1}}\left(\boldsymbol{R}, \boldsymbol{R}_{1}\right)=\left[B_{\sigma}(\boldsymbol{R}),\left[B_{\sigma_{1}}\left(\boldsymbol{R}_{1}\right), H_{S C}\right]\right]$. In Eq. (3) we have introduced the linear optical response $p_{\sigma}(\boldsymbol{R}, t)$. The latter is governed by an equation of the same form as Eq. (2) but with the source term $F_{\sigma}^{(1)}(\boldsymbol{R}, t)=\boldsymbol{d}_{\sigma} \cdot \boldsymbol{E}(\boldsymbol{R}, t)$, where $\boldsymbol{d}$ is the dipole moment and $E$ is the electric field of the excitation. Taking the energy of the $\mathrm{HH}$ exciton in the perfect QW as zero, the Hamiltonian in Eq. (2) can be written as $\hat{H}_{\sigma}(\boldsymbol{R})=$ $-\frac{1}{2 M_{\sigma}} \nabla_{\boldsymbol{R}}^{2}+W_{\sigma}(\boldsymbol{R})$ with

$$
\begin{aligned}
W_{\sigma}(\boldsymbol{R})= & \int d \rho\left|\psi_{\sigma}(\boldsymbol{\rho})\right|^{2} \\
& \times\left[\delta E_{e}\left(\boldsymbol{R}+\frac{\rho m_{\sigma}}{M_{\sigma}}\right)+\delta E_{\sigma}\left(\boldsymbol{R}-\frac{\rho m_{s}}{M_{\sigma}}\right)\right],
\end{aligned}
$$

where $\delta E_{e, \sigma}(\boldsymbol{R})$ are local variations of the electron and hole energies due to fluctuations of the width of the QW. The convolution with the exciton wave function results in smoothing the spatial variation of the disorder potential on the scale of the exciton Bohr radius. For our analysis, however, we use a simplified model for $W_{\sigma}(\boldsymbol{R})$, presenting it as $W_{\sigma}(\boldsymbol{R})=$ $\sum_{m} w_{\sigma}^{(m)}\left(\boldsymbol{R}-\boldsymbol{r}_{m}\right)$, where $\boldsymbol{r}_{m}$ is the position of $m$ th defect and $w_{\sigma}^{(m)}(\boldsymbol{r})=V_{\sigma}^{(m)}$, if $|\boldsymbol{r}|<r_{d}$ and 0 , otherwise with $V_{\sigma}^{(m)}>0$ and $V_{\sigma}^{(m)}<0$ for NW and WW defects, respectively. Such approximation is justified for the system under consideration by the fact that the exciton resonances are split. The size of the defects must exceed the lateral confinement length $\xi_{\sigma}=\pi / \sqrt{2 M_{\sigma} V_{\sigma}}$, which, in turn, has to be larger than the Bohr radius in order for such multiplets to form. ${ }^{1}$ Therefore, the effect of spatial features of the order of the Bohr radius on optical response is weak.

Equation (2) yields a detailed description of the nonlinear response of disordered, inhomogeneous QWs. It should be noted that the spatial distributions of linear and nonlinear responses are very complex owing to broken in-plane translational symmetry. The picture simplifies drastically, however, if one is interested in the response along phasematching directions only. If inhomogeneities have the same statistical characteristics across the QW, the responses exhibit singularities along the phase matching direction similarly to an ideal QW. The respective amplitudes can be shown to be determined by the statistical average of the total response. Thus, in order to simulate the $2 \mathrm{D}$ spectrum, one only needs to average $P_{\sigma}^{(3)}(\boldsymbol{R}, t)$ over statistics of the inhomogeneities and take the respective Fourier transforms.

The model above is still quite general; we make additional simplifications in order to describe main features of the experimental spectrum. We limit ourselves to consider only $\mathrm{HH}$ excitons with the definite spin state determined by the polarization of the external excitation, and we take the limit of zero waiting time (e.g., neglecting incoherent dynamics) and assume that the nonlinear response along the phase-matching direction weakly depends on small in-plane projections of the wave vectors of the excitation pulses. Applying these assumptions we find the response along the FWM direction corresponding to the rephasing scheme in the form

$$
\begin{aligned}
& P^{(F W M)}\left(\omega_{t}, \omega_{\tau}\right) \\
& =-i \int d \omega_{1} d \omega_{2} d \omega_{3} \delta\left(\omega_{t}+\omega_{1}-\omega_{2}-\omega_{3}\right) \\
& \quad \times \frac{1}{\omega_{\tau}+\omega_{1}} f_{1}^{*}\left(\omega_{1}\right) f_{2}\left(\omega_{2}\right) f_{3}\left(\omega_{3}\right) \Pi\left(\omega_{t}, \omega_{1}, \omega_{2}, \omega_{3}\right),
\end{aligned}
$$


where $f_{j}(\omega)$ is the amplitude of the $j$ th excitation pulse, $j=$ $1,2,3$, and

$$
\begin{aligned}
\Pi\left(\omega_{t}, \omega_{1}, \omega_{2}, \omega_{3}\right) \\
=\frac{1}{S} \int d \boldsymbol{R} \int d \boldsymbol{R}_{1} d \boldsymbol{R}_{2} d \boldsymbol{R}_{3}\left\langle\beta\left(\boldsymbol{R}, \boldsymbol{R}_{1}, \boldsymbol{R}_{2}, \boldsymbol{R}_{3}\right)\right. \\
\left.\quad \times p^{*}\left(\boldsymbol{R}, \omega_{t}\right) p^{*}\left(\boldsymbol{R}_{1}, \omega_{1}\right) p\left(\boldsymbol{R}_{2}, \omega_{2}\right) p\left(\boldsymbol{R}_{3}, \omega_{3}\right)\right\rangle .
\end{aligned}
$$

Here, the integral over $\boldsymbol{R}$ is assumed to be taken over the QW area $S$ with the subsequent limit $S \rightarrow \infty$, and linear responses $p(\boldsymbol{R}, \omega)$ are found assuming the unit magnitude of the external excitation.

Considering only the effect of disorder on the linear response functions, the $2 \mathrm{D}$ spectrum is determined by the statistical average of the product of linear response functions corresponding to four frequencies taken at four points. Such correlators are similar to those investigated in the theory of transport in disordered media in the context of speckle patterns. ${ }^{46-48}$ This demonstrates that the 2D spectroscopy is sensitive to nontrivial correlation properties of excitations in disordered media, and therefore it is necessary to distinguish between two different contributions to the 2D spectrum: the local interaction between states corresponding to different frequencies and coupling between excitons in spatially separated regions of the $\mathrm{QW}$ provided by the effective potential $\beta$. The first contribution is similar to what one has in the case of homogeneous QWs, while the second is specific to systems with broken translational symmetry by excitation or disorder. The relation between these contributions is determined by the relation between length scales of the spatial decay of the effective potential and the correlator of linear responses. In Ref. 39, a simple case was considered when the main effect is due to the spatial extent of $\beta$. Expanding Eq. (4) in terms of the electron and hole wave functions, one can see that $\beta$ consists essentially of two parts accounting for the interaction of the Van der Waals type and for the exchange by electrons and holes between excitons. Both contributions are important at the scale of the Bohr radius, while at larger distances the Van der Waals part decays $\propto 1 / r^{6}$, and the exchange contribution decays exponentially. In the context of describing coherent coupling among excition resonances, we can approximate the effective potential by a contact interaction: $\beta\left(\boldsymbol{R}, \boldsymbol{R}_{1}, \boldsymbol{R}_{2}, \boldsymbol{R}_{3}\right)=$ $\beta \delta\left(\boldsymbol{R}-\boldsymbol{R}_{1}\right) \delta\left(\boldsymbol{R}-\boldsymbol{R}_{2}\right) \delta\left(\boldsymbol{R}-\boldsymbol{R}_{3}\right)$ because both the Van der Waals and exchange interactions act on length scales smaller than the disorder correlation length, i.e., the average lateral size of disorder potential. Using this approximation and integrating over $\boldsymbol{R}_{1,2,3}$, we express the 2D spectrum in terms of the spectral correlator calculated at point $\boldsymbol{R}$. Note that in this case, the statistical averaging becomes redundant and can be omitted. Furthermore, the correlator can be expanded in terms of contributions of individual defects using the Korringa-Kohn-Rostoker Green-function method. ${ }^{49}$ Taking into account the low density of the defects, which is evidenced by the low intensity of type $A$ and $C$ exciton resonances in photoluminescence measurements (data not shown), we can neglect the effect of the multiple scattering and obtain

$$
\begin{aligned}
& \Pi\left(\omega_{t}, \omega_{1}, \omega_{2}, \omega_{3}\right) \\
& =\sum_{i} n_{i} \beta \int_{S_{d}} d \boldsymbol{R} p_{i}^{*}(\boldsymbol{R}, \omega) p_{i}^{*}\left(\boldsymbol{R}, \omega_{1}\right) p_{i}\left(\boldsymbol{R}, \omega_{2}\right) p_{i}\left(\boldsymbol{R}, \omega_{3}\right),
\end{aligned}
$$

where $i$ enumerates types of defects (in the case under consideration, they are NW and WW defects), $n_{i}$ is the density of the defects of the respective type, $p_{i}(\boldsymbol{R}, \omega)$ is the linear response of a $\mathrm{QW}$ with a single defect, and the integral is taken over the area per one defect $S_{d}=1 / \sum_{i} n_{i}$.

Owing to the complex frequency dependence of the linear response, the $2 \mathrm{D}$ spectrum has, in general, a rich structure. However, since the resonant frequencies are well separated, we can simplify it even further. Using a resonant expansion ${ }^{50,51}$ for the linear response and retaining only main resonant contributions to the $2 \mathrm{D}$ spectrum, we find

$$
P_{F W M}^{(3)}\left(\omega_{t}, \omega_{\tau}\right) \approx \sum_{i, j} \frac{\alpha_{i, j}}{\left(\omega_{\tau}+\omega_{i}-i \gamma_{i}\right)\left(\omega_{t}-\omega_{j}+i \gamma_{j}\right)\left(\omega_{t}-\omega_{j}+2 i \gamma_{i}+i \gamma_{j}\right)},
$$

where $i$ and $j$ are the indices enumerating different resonances $\{A, B, C\}$, and the overlap parameters are $\alpha_{i, j} \sim$ $n \beta \int d \boldsymbol{R}\left|p\left(\boldsymbol{R}, \omega_{i}\right)\right|^{2}\left|p\left(\boldsymbol{R}, \omega_{j}\right)\right|^{2}$. It should be noted that due to the permutation of integrations over coordinates and frequencies, special care should be taken of the contribution due to the scattering resonance because it is described by non-Hermitian Gamow vectors. ${ }^{51,52}$ In this case, $|p(\boldsymbol{R}, \omega)|^{2} \equiv$ $\overline{p(\boldsymbol{R}, \omega)} p(\boldsymbol{R}, \omega)$, where $\overline{p(\boldsymbol{R}, \omega)}$ is a conjugate solution (i.e., satisfying the incoming boundary conditions at infinity).

The obtained expression for the 2D spectrum clearly demonstrates its main features. Exciton linear response functions $p\left(\boldsymbol{R}, \omega_{i}\right)$ vary significantly depending on the type of excitons. To illustrate this point, we performed a simple calculation of linear response near a single circular defect in an otherwise perfect QW. The calculated linear response near WW and NW defects at corresponding resonant frequencies are shown in Figs. 4(a) and 4(b), respectively. Type $A$ excitons [solid red line in Fig. 4(a)] correspond to bound states that are localized within WW defects and decay exponentially outside. This fast spatial decay leads to small overlap with type $B$ excitons [dashed green curves in Figs. 4(a) and 4(b)] and even less overlap with type $C$ excitons [solid blue curve in Fig. 4(b)] due to the small spatial density of the defects. In contrast, type $C$ excitons are unbounded scattering resonances that decay algebraically outside of NW defects. Type $B$ excitons largely occupy the perfect $\mathrm{QW}$ region, but their linear response functions are perturbed near either type of defects. The strength of the cross peaks is determined by $\alpha_{i, j}$. Qualitatively, the large 

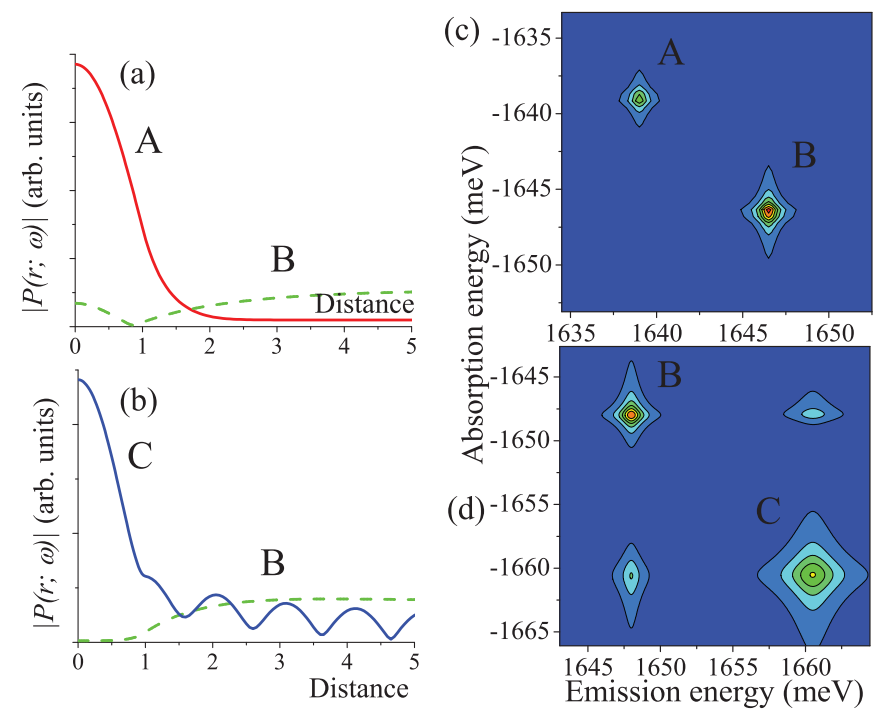

FIG. 4. (Color online) Calculated linear response function vs distance (normalized to the radius of the defect) to the center of a single circular defect for (a) WW disorder and (b) NW disorder. Green dashed lines are for type $B$ excitons. Solid red line is for type $A$ excitons in (a), and solid blue line is for type $C$ excitons in (b). Simulated 2D spectra when (c) type $A$ and type $B$ excitons are excited and (d) type $B$ and type $C$ excitons are excited. The linewidth of exciton resonances are chosen to be $1 \mathrm{meV}$ in (c) and (d).

spatial overlap between excitons $B$ and $C$ leads to coherent coupling, while the minimal overlap between excitons $A$ and $B$ accounts for the lack of coupling as illustrated by Figs. 4(a) and 4(b).
Simulated 2D spectra are shown in Figs. 4(c) and 4(d), where the latter confirm coherent coupling between exciton types $B$ and $C$. The calculations neglected the incoherent population relaxation dynamics and included several parameters, including dephasing times and overlap factors $\left(\alpha_{A B}, \alpha_{B C}\right.$, and $\alpha_{A C}$ ) determined from the single circular defect calculations. Although the simulated spectra cannot make a quantitative prediction of coupling strength due to the lack of detailed information on the disorder potential, the qualitative features on the presence or absence of coherent coupling are reproduced robustly.

\section{SUMMARY}

In summary, we have identified different types of exciton resonances in a disordered $\mathrm{QW}$, and more importantly, articulated how the disorder potential governs coherent coupling between these excitons. Our findings may be applicable to a broad range of problems, including energy transfer in natural/artificial photosynthetic systems ${ }^{13-15}$ and charge transfer in conjugated polymers.

\section{ACKNOWLEDGMENTS}

Authors at the University of Texas, Austin, gratefully acknowledge financial support from the following sources: National Science Foundation (NSF) DMR-0747822, Welch Foundation F-1662, ARO-W911NF-08-1-0348, and the Alfred P. Sloan Foundation. M.N.L. acknowledges support from NSF (Grant No. ECCS-0901784), AFOSR (Grant No. FA9550-091-0450), and NSF (Grant No. ECCS-1128597).
*Corresponding author: elaineli@physics.utexas.edu

${ }^{1}$ H. Castella and J. W. Wilkins, Phys. Rev. B 58, 16186 (1998).

${ }^{2}$ V. Savona and W. Langbein, Phys. Rev. B 74, 075311 (2006).

${ }^{3}$ D. Bimberg, J. Christen, T. Fukunaga, H. Nakashima, D. E. Mars, and J. N. Miller, J. Vac. Sci. Technol. B 5, 1191 (1987).

${ }^{4}$ D. Gammon, B. V. Shanabrook, and D. S. Katzer, Phys. Rev. Lett. 67, 1547 (1991).

${ }^{5}$ E. O. Göbel, K. Leo, T. C. Damen, J. Shah, S. Schmitt-Rink, W. Schäfer, J. F. Müller, and K. Köhler, Phys. Rev. Lett. 64, 1801 (1990).

${ }^{6}$ M. Koch, J. Feldmann, E. O. Göbel, P. Thomas, J. Shah, and K. Kohler, Phys. Rev. B 48, 11480 (1993).

${ }^{7}$ A. Euteneuer, E. Finger, M. Hofmann, W. Stolz, T. Meier, P. Thomas, S. W. Koch, W. W. Ruhle, R. Hey, and K. Ploog, Phys. Rev. Lett. 83, 2073 (1999).

${ }^{8}$ M. Phillips and H. L. Wang, Solid State Commun. 111, 317 (1999).

${ }^{9}$ J. Kasprzak, B. Patton, V. Savona, and W. Langbein, Nat. Photonics 5, 57 (2011).

${ }^{10}$ T. Takagahara, Phys. Rev. B 31, 6552 (1985).

${ }^{11}$ R. Singh and G. Bester, Phys. Rev. Lett. 103, 063601 (2009).

${ }^{12}$ P. C. Chen, C. Piermarocchi, L. J. Sham, D. Gammon, and D. G. Steel, Phys. Rev. B 69, 075320 (2004).
${ }^{13}$ G. S. Engel, T. R. Calhoun, E. L. Read, T. K. Ahn, T. Mancal, Y. C. Cheng, R. E. Blankenship, and G. R. Fleming, Nature (London) 446, 782 (2007).

${ }^{14}$ A. Ishizaki and G. R. Fleming, J. Phys. Chem. B 115, 6227 (2011).

${ }^{15}$ E. Collini, C. Y. Wong, K. E. Wilk, P. M. G. Curmi, P. Brumer, and G. D. Scholes, Nature (London) 463, 644 (2010).

${ }^{16} \mathrm{M}$. Pope and C. E. Swenberg, Electronic Processes in Organic Crystals and Polymers (Oxford University Press, New York, 1999).

${ }^{17}$ C. L. Braun, J. Chem. Phys. 80, 4157 (1984).

${ }^{18}$ X. Li, T. Zhang, C. N. Borca, and S. T. Cundiff, Phys. Rev. Lett. 96, 057406 (2006)

${ }^{19}$ S. Mukamel, Annu. Rev. Phys. Chem. 51, 691 (2000).

${ }^{20}$ M. Cho, Chem. Rev. 108, 1331 (2008).

${ }^{21}$ I. Kuznetsova, N. Gogh, J. Forstner, T. Meier, S. T. Cundiff, I. Varga, and P. Thomas, Phys. Rev. B 81, 075307 (2010).

${ }^{22}$ A. D. Bristow, T. H. Zhang, M. E. Siemens, S. T. Cundiff, and R. P. Mirin, J. Phys. Chem. B 115, 5365 (2011).

${ }^{23}$ J. A. Davis, C. R. Hall, L. V. Dao, K. A. Nugent, H. M. Quiney, H. H. Tan, and C. Jagadish, J. Chem. Phys. 135, 044510 (2011).

${ }^{24}$ A. D. Bristow, D. Karaiskaj, X. Dai, T. Zhang, C. Carlsson, K. R. Hagen, R. Jimenez, and S. T. Cundiff, Rev. Sci. Instrum. 80, 073108 (2009).

${ }^{25}$ H. Wang, K. Ferrio, D. G. Steel, Y. Z. Hu, R. Binder, and S. W. Koch, Phys. Rev. Lett. 71, 1261 (1993). 
${ }^{26}$ Y. Z. Hu, R. Binder, S. W. Koch, S. T. Cundiff, H. Wang, and D. G. Steel, Phys. Rev. B 49, 14382 (1994).

${ }^{27}$ P. V. Santos, M. Willatzen, M. Cardona, and A. Cantarero, Phys. Rev. B 51, 5121 (1995).

${ }^{28}$ D. Gammon, E. S. Snow, B. V. Shanabrook, D. S. Katzer, and D. Park, Phys. Rev. Lett. 76, 3005 (1996).

${ }^{29}$ A. S. Bracker, E. A. Stinaff, D. Gammon, M. E. Ware, J. G. Tischler, D. Park, A. V. Filinov, M. Bonitz, F. Peeters, and C. Riva, Phys. Rev. B 72, 035332 (2005).

${ }^{30} \mathrm{See}$ Supplemental Material at http://link.aps.org/supplemental/ 10.1103/PhysRevB.88.075316 for more characterization measurements.

${ }^{31} \mathrm{We}$ performed 2DFTS experiments on all QWs and observed systematic changes in the cross peaks. For example, no coupling was observed between any $\mathrm{HH}$ excitons in the widest QW. Coupling between $\mathrm{HH}-\mathrm{LH}$ in different regions was observed in the QW with the intermediate thickness. It is outside the scope of the current paper to present results from all QWs.

${ }^{32}$ C. N. Borca, T. H. Zhang, X. Q. Li, and S. T. Cundiff, Chem. Phys. Lett. 416, 311 (2005).

${ }^{33}$ This statement is true under the condition that both dipole transitions are excited by equal electric field strength.

${ }^{34}$ G. Moody, M. E. Siemens, A. D. Bristow, X. Dai, A. S. Bracker, D. Gammon, and S. T. Cundiff, Phys. Rev. B 83, 245316 (2011).

${ }^{35}$ K. W. Stone, K. Gundogdu, D. B. Turner, X. Q. Li, S. T. Cundiff, and K. A. Nelson, Science 324, 1169 (2009).

${ }^{36}$ D. Karaiskaj, A. D. Bristow, L. J. Yang, X. C. Dai, R. P. Mirin, S. Mukamel, and S. T. Cundiff, Phys. Rev. Lett. 104, 117401 (2010).

${ }^{37}$ L. Yang and S. Mukamel, Phys. Rev. Lett. 100, 057402 (2008).
${ }^{38}$ T. Östreich, K. Schönhammer, and L. J. Sham, Phys. Rev. B 58, 12920 (1998).

${ }^{39}$ M. Erementchouk, M. N. Leuenberger, and X. Q. Li, Phys. Status Solidi C 8, 1141 (2011).

${ }^{40}$ R. Zimmermann and E. Runge, Phys. Status Solidi A 164, 511 (1997).

${ }^{41}$ M. Lindberg, Y. Z. Hu, R. Binder, and S. W. Koch, Phys. Rev. B 50, 18060 (1994).

${ }^{42}$ K. Victor, V. M. Axt, and A. Stahl, Phys. Rev. B 51, 14164 (1995).

${ }^{43}$ W. Schäfer, D. S. Kim, J. Shah, T. C. Damen, J. E. Cunningham, K. W. Goossen, L. N. Pfeiffer, and K. Köhler, Phys. Rev. B 53, 16429 (1996).

${ }^{44}$ N. H. Kwong, R. Takayama, I. Rumyantsev, M. Kuwata-Gonokami, and R. Binder, Phys. Rev. B 64, 045316 (2001).

${ }^{45}$ S. Savasta, O. Di Stefano, and R. Girlanda, Phys. Rev. Lett. 90, 096403 (2003).

${ }^{46}$ M. C. W. van Rossum and T. M. Nieuwenhuizen, Rev. Mod. Phys. 71, 313 (1999).

${ }^{47}$ O. Agam, A. V. Andreev, and B. Spivak, Phys. Rev. Lett. 97, 223901 (2006).

${ }^{48}$ P. Sebbah, R. Pnini, and A. Z. Genack, Phys. Rev. E 62, 7348 (2000).

${ }^{49}$ W. Kohn and N. Rostoker, Phys. Rev. 94, 1111 (1954).

${ }^{50}$ P. D. Lax and R. S. Phillips, Scattering Theory (Academic Press, San Diego, 1989).

${ }^{51}$ R. de la Madrid, G. Garcia-Calderon, and J. G. Muga, Czech. J. Phys. 55, 1141 (2005).

${ }^{52}$ P. L. Garrido, S. Goldstein, J. Lukkarinen, and R. Tumulka, Am. J. Phys. 79, 1218 (2011). 